\title{
OPEN Cell-fate decision of mesenchymal stem cells toward osteocyte differentiation is committed by spheroid culture
}

\author{
Jeonghyun $\mathrm{Kim}^{1,2 \rrbracket}$ \& Taiji Adachi $^{1}$
}

Osteocytes are mechanosensory commander cells to regulate bone remodeling throughout the lifespan. While the osteocytes are known as terminally differentiated cells derived from mesenchymal stem cells, the detailed mechanisms of osteocyte differentiation remain unclear. In this study, we fabricated 3D self-organized spheroids using human mesenchymal stem cells (MSCs). Under the osteogenesis induction medium, the spheroid culture model exerted the osteocyte-likeness within 2 days compared to a conventional 2D monolayer model. Moreover, we showed that an inhibition of actin polymerization in the spheroid further up-regulated the osteocyte gene expressions. Notably, we represented that the cell condensed condition acquired in the 3D spheroid culture model determined a differentiation fate of MSCs to osteocytes. Taken together, we suggest that our selforganized spheroid model can be utilized as a new in vitro model to represent the osteocyte and to recapitulate an in vitro ossification process.

The bone undertakes continuous remodeling by osteoclasts and osteoblasts throughout the lifespan while its balance is modulated by mechanosensory commander cells, osteocytes ${ }^{1,2}$. From the view of the developmental process, the ossification is initiated by mesenchymal condensation. In other words, the osteocyte is also known as a terminally differentiated bone cell derived from the mesenchymal stem cells (MSCs). The MSCs are referred to as multipluripotent progenitor cells capable of differentiation into skeletal tissues such as osteoblasts, chondrocytes, adipocytes, myocytes ${ }^{3-5}$. After the osteoblasts produce the bone matrices such as collagen type I and alkaline phosphatase during the bone remodeling process ${ }^{6,7}$, it is known that they encounter one of the fates; (1) to become bone-lining cells, (2) to become osteocytes embedded in the bone, and (3) to undertake apoptosis ${ }^{2,8}$.

For both osteoblast differentiation and bone mineralization studies, many researchers have established a gold standard protocol to induce in vitro osteogenesis using chemical osteogenesis induction (OI) supplements such as ascorbic acid and $\beta$-glycerophosphate ${ }^{9,10}$. On the other hand, in vitro osteocyte differentiation has been also attempted by many studies. After the osteoblast precursor cells were subjected to a long period of cultivation in the OI culture medium on the culture dish, a three-dimensional dome-shape of the bone-like nodule was formed ${ }^{11-13}$. Although the osteocyte-like cells were eventually observed inside the nodule after several weeks, the efficient method to achieve the direct osteocyte differentiation derived from pre-osteoblast cells or stem cells has not been established since the osteocyte-like cells were randomly shown in the nodule after such a long cultivation. Moreover, it still remains difficult for primary osteocytes to culture in vitro and further to maintain the cellular morphology and functions for a long period of time, so that a new in vitro osteocyte model is required to be established with a novel efficient method. After achieving this, it will help to unravel the unknown mechanisms of cell-fate decision toward osteocytes.

In previous studies, our group has elucidated the effect of three-dimensional culture for pre-osteoblast cells on the osteocyte differentiation ${ }^{14-16}$. Compared to the conventional monolayer model, the pre-osteoblast cells in the form of scaffold-free spheroid model rendered the osteocyte-likeness within 2 days in the absence of the OI supplements. In this study, we fabricated the 3D spheroid culture model reconstructed by the MSCs and evaluated its osteocyte differentiation capability. By using the MSC spheroid model, we attempted to elucidate the mechanism of the cell-fate decision of MSCs toward osteocytes and also to recapitulate the in vitro ossification process derived from the mesenchymal condensation. 
(A)

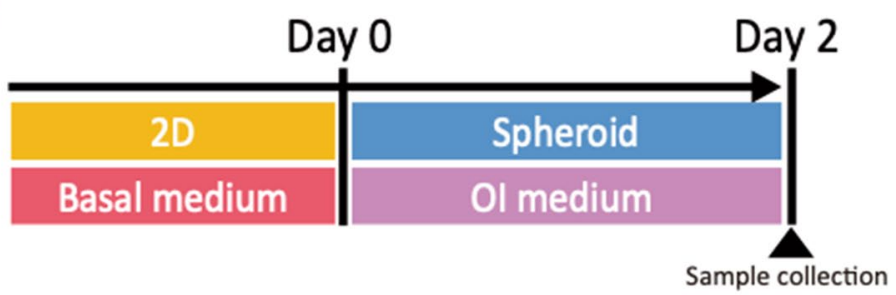

(C)
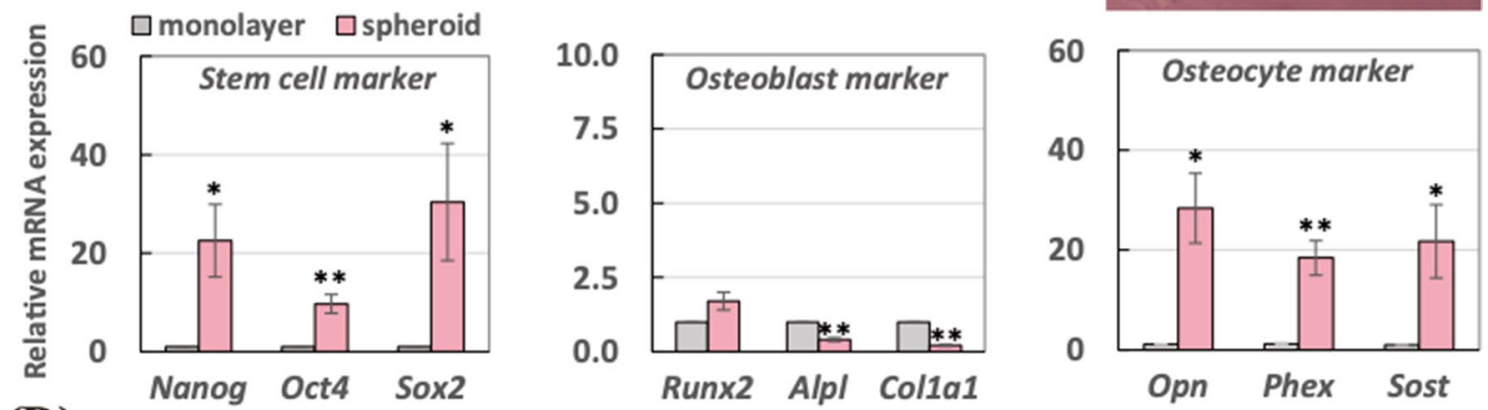

(D)
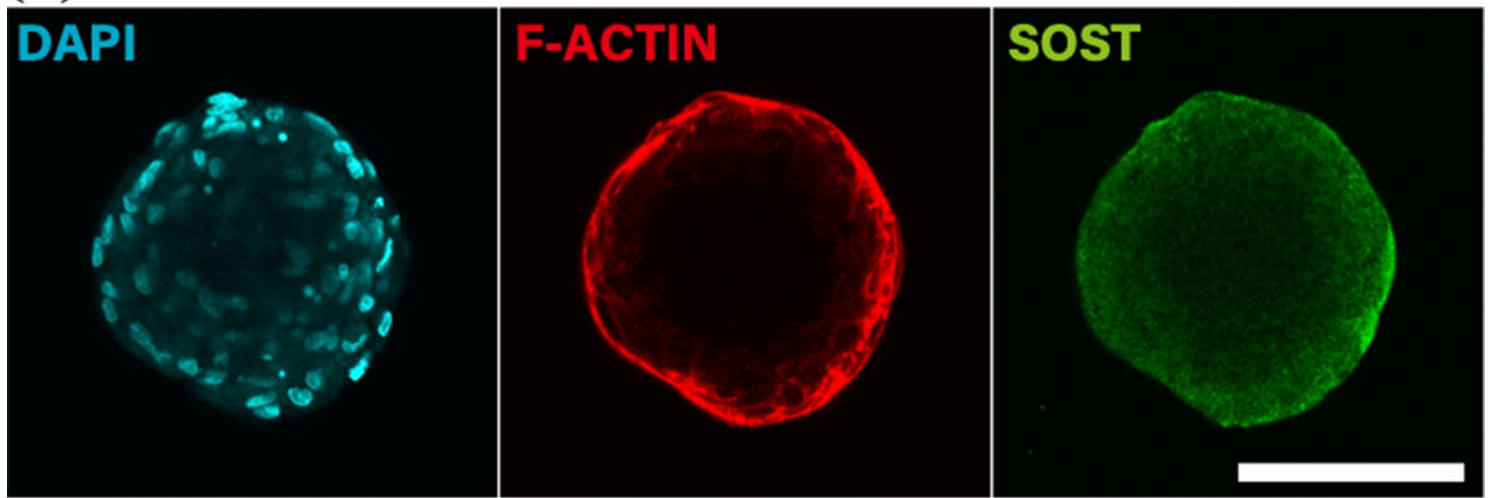

Figure 1. (A) Schematic of the experimental time line for spheroids incubated in the osteogenesis induction medium for 2 days. (B) Morphology of the spheroid after a 2-day incubation period. The scale bar represents $100 \mu \mathrm{m}$. (C) Relative mRNA expressions of stem cell markers (Nanog, Oct4, and Sox2), osteoblast markers (Runx2, Alpl, and Col1a1), and osteocyte markers (Opn, Phex, and Sost) were measured by real-time PCR. All the mRNA expressions were normalized to Gapdh expressions while the results were expressed as relative amounts against the expression of monolayer sample $(n=8)$. The bars represent the mean \pm standard error. $P$-value was calculated from Student's $t$-test; ${ }^{\star} p<0.05$, ${ }^{* *} p<0.005$. (D) Immunostaining images of spheroid after 2-day incubation; cell nuclei (DAPI in cyan), actin filaments (F-Actin in red), and sclerostin (SOST in green). The scale bar represents $100 \mu \mathrm{m}$.

\section{Results}

MSC spheroid with osteogenic induction supplements up-regulated osteocyte markers within 2 days. As described in Fig. 1A, the spheroids reconstructed by hMSCs were incubated in the osteogenic induction (OI) medium for 2 days. In Fig. 1B, the spheroid has about $185 \mu \mathrm{m}$ diameter at 2 -day. To evaluate gene expression changes in the spheroid compared to the monolayer, we carried out real-time PCR in Fig. 1C. After 2-day incubation, the relative mRNA expression in the spheroid for stem cell markers were up-regulated compared to that in the monolayer; Nanog (22.6-fold change; $p<0.05)$, Oct4 (9.7-fold change; $p<0.005$ ), and Sox2 (30.4-fold change; $p<0.05$ ). Regarding osteoblast markers, there was no significant mRNA expression change in Runx2 (1.70-fold change), while both Alpl $(0.41$-fold change; $p<0.005)$ and Colla1 $(0.21$-fold change; $p<0.005)$ mRNA expressions were down-regulated in the spheroid. On the other hand, the relative mRNA expressions for osteocyte markers in the spheroids compared to the monolayer were highly up-regulated; Opn (28.4-fold change; $p<0.05)$, Phex (18.4-fold change; $p<0.005$ ), and Sost $(21.7$-fold change; $p<0.05)$. To examine the protein level expression of the osteocyte marker, we conducted the SOST immunostaining in Fig. 1D since the SOST is known as a marker of mature osteocytes. As a result, the SOST expression (green) in the protein level was detected entirely inside the spheroid after 2-day incubation. Moreover, F-actin was expressed strongly on the surface of the spheroid compared to the inner part. 
(A)

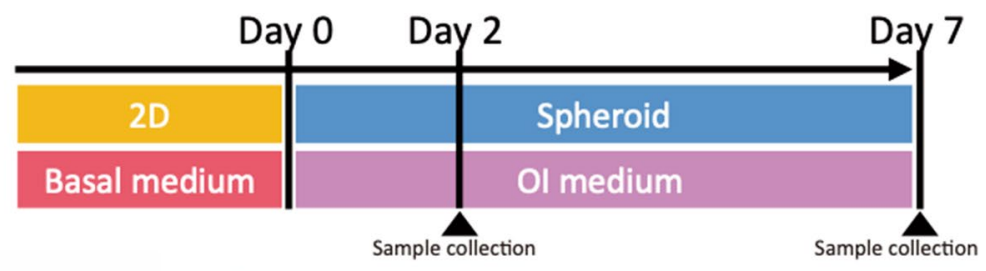

(C)

(D)
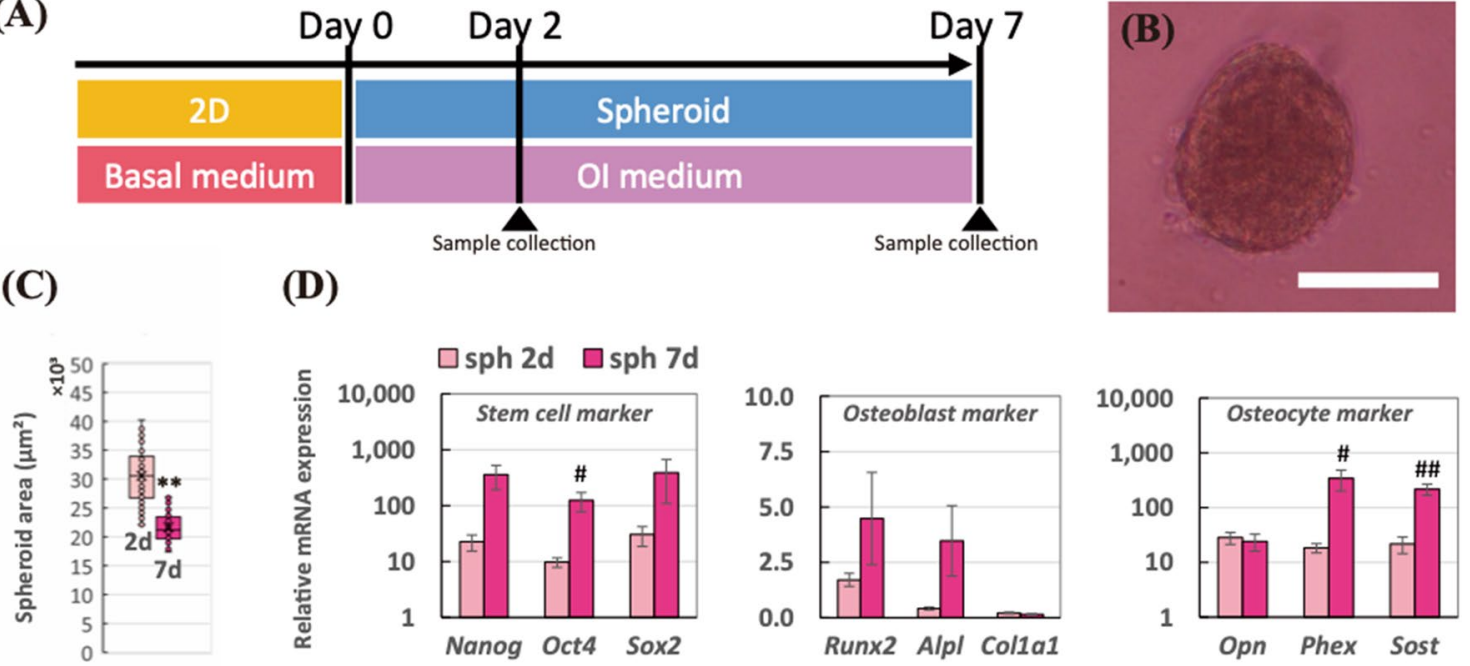

(E)
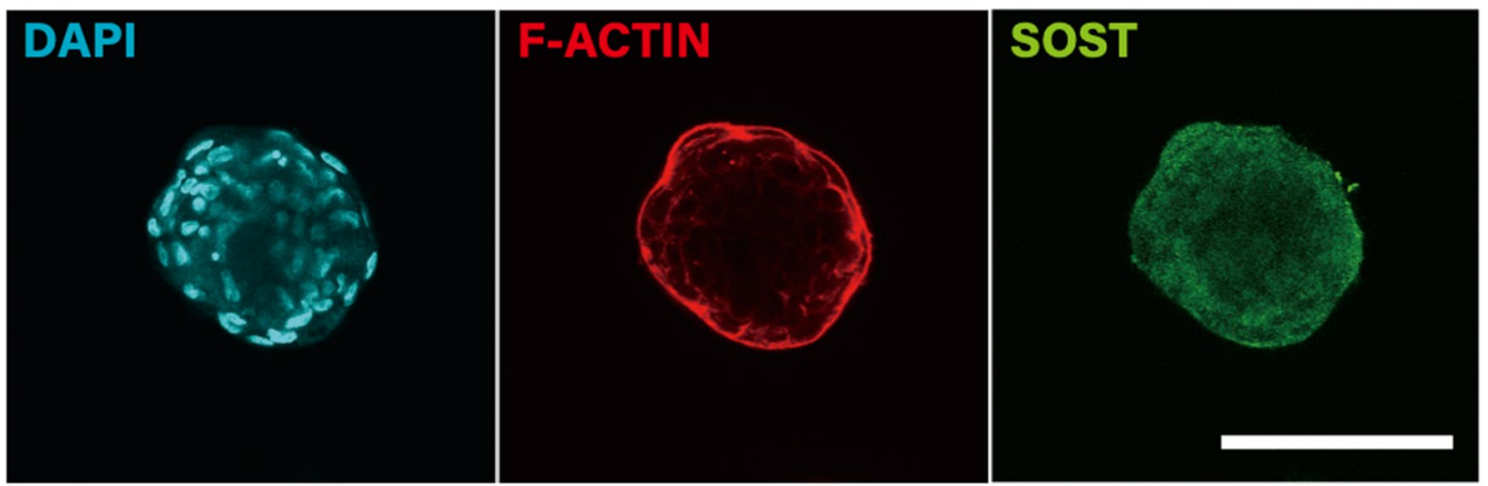

Figure 2. (A) Schematic of the experimental time line for spheroids incubated in the osteogenesis induction medium for 7 days. (B) Morphology of the spheroid after a 7-day incubation period. The scale bar represents $100 \mu \mathrm{m}$. (C) Mean values of projected area of the spheroids after 2 and 7 days. F-test was performed to examine the significance of area changes in spheroids ( $n=60$ from 5 independent experiments; $\left.{ }^{*} p<0.05,{ }^{* *} p<0.005\right)$. (D) Relative mRNA expressions of stem cell markers (Nanog, Oct4, and Sox2), osteoblast markers (Runx2, Alpl, and Col1a1), and osteocyte markers (Opn, Phex, and Sost) in the spheroids at 2-day and 7-day incubation were measured by real-time PCR. All the mRNA expressions were normalized to Gapdh expressions while the results were expressed as relative amounts against the expression of monolayer sample $(n=8)$. The bars represent the mean \pm standard error. $P$-value was calculated from Student's $t$-test; ${ }^{\#} p<0.05$, \#\# $p<0.005$. (E) Immunostaining images of spheroid after 2-day incubation; cell nuclei (DAPI in cyan), actin filaments (F-actin in red), and sclerostin (SOST in green). The scale bar represents $100 \mu \mathrm{m}$.

Up-regulated osteocyte markers in the spheroid were prolonged after 7 days. To investigate the long-term persistence of gene expressions for osteocyte markers in the spheroid, we performed the longer experiment, 7-day, as shown in Fig. 2A. The spheroid at 7-day in Fig. 2B became smaller compared to 2-day spheroid $(48.9 \%$ reduction; $p<0.005)$. In Fig. $2 \mathrm{C}$, the mean values of the projected area of the spheroid at 2-day and 7-day were 25,900 $\mu^{2}$ and 12,700 $\mu \mathrm{m}$ Department of Mechanical Systems Engineering, Nagoya University, Nagoya 464-8603, Japan, respectively. In Fig. 2D, we conducted real-time PCR to examine the gene expression changes between 2-day and 7-day spheroid. Consequently, the stem cell markers in the 7-day spheroid were upregulated compared to the 2-day spheroid; Nanog (15.9-fold change; $p=0.07)$, Oct4 (12.8-fold change; $p<0.05$ ), and Sox2 (12.7-fold change; $p=0.26)$. Osteoblast markers were non-significantly modulated; Runx2 (2.63-fold change; $p=0.18)$, Alpl (8.44-fold change; $p=0.09)$, and Colla1 (0.68-fold change; $p=0.12)$. Regarding osteocyte markers, while early osteocyte marker, $O p n(0.86$-fold change; $p=0.75)$, was not modulated, it up-regulated the mature osteocyte markers in the 7-day spheroid including Phex (18.5-fold change; $p<0.05)$ and Sost $(10.0$ fold change; $p<0.005)$. Immunostaining results in Fig. $2 \mathrm{E}$ represented that the SOST expression was entirely expressed in the 7-day spheroid.

Structural effect achieved in 3D spheroid compared to 2D monolayer promoted stem cell, osteocyte, and hypertrophic chondrocyte markers. As described in Fig. 3A, the spheroids were incubated in the basal medium in the absence of OI medium to elucidate the structural effect achieved in the 3D spheroid culture compared to the $2 \mathrm{D}$ monolayer condition, without the chemical supplements. In Fig. 3B, we measured the gene expression changes in stem cell, osteoblast, and osteocyte markers. As a result, all the stem 

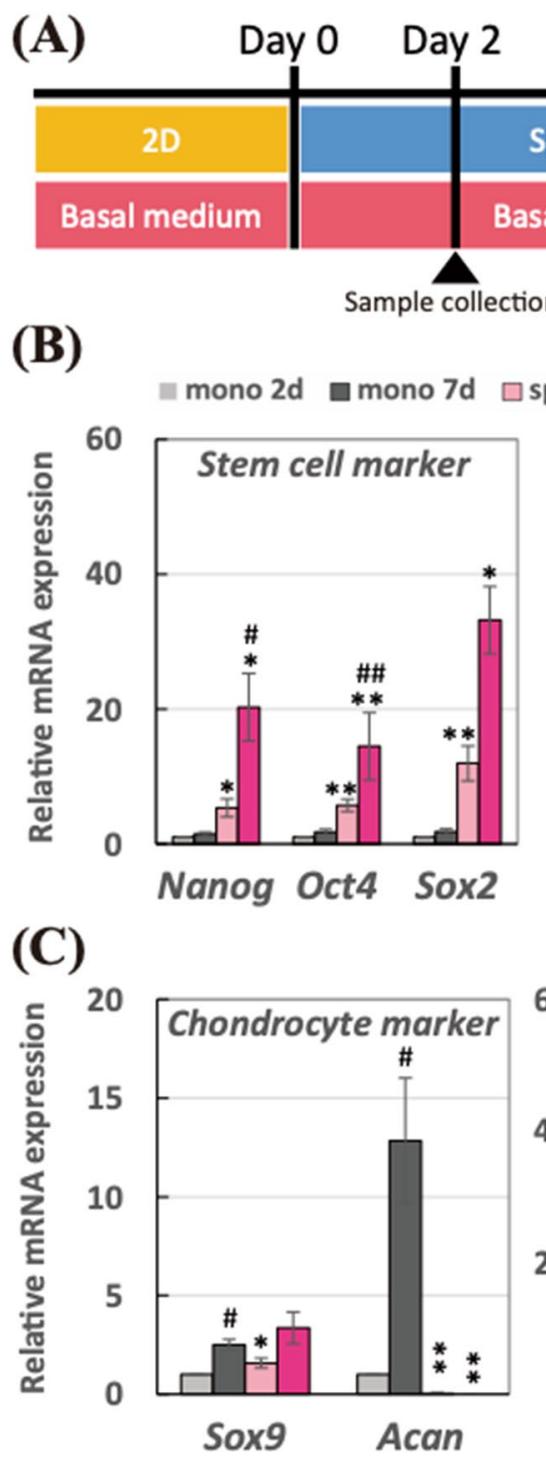

Spheroid

Basal medium

tion

Sample collection
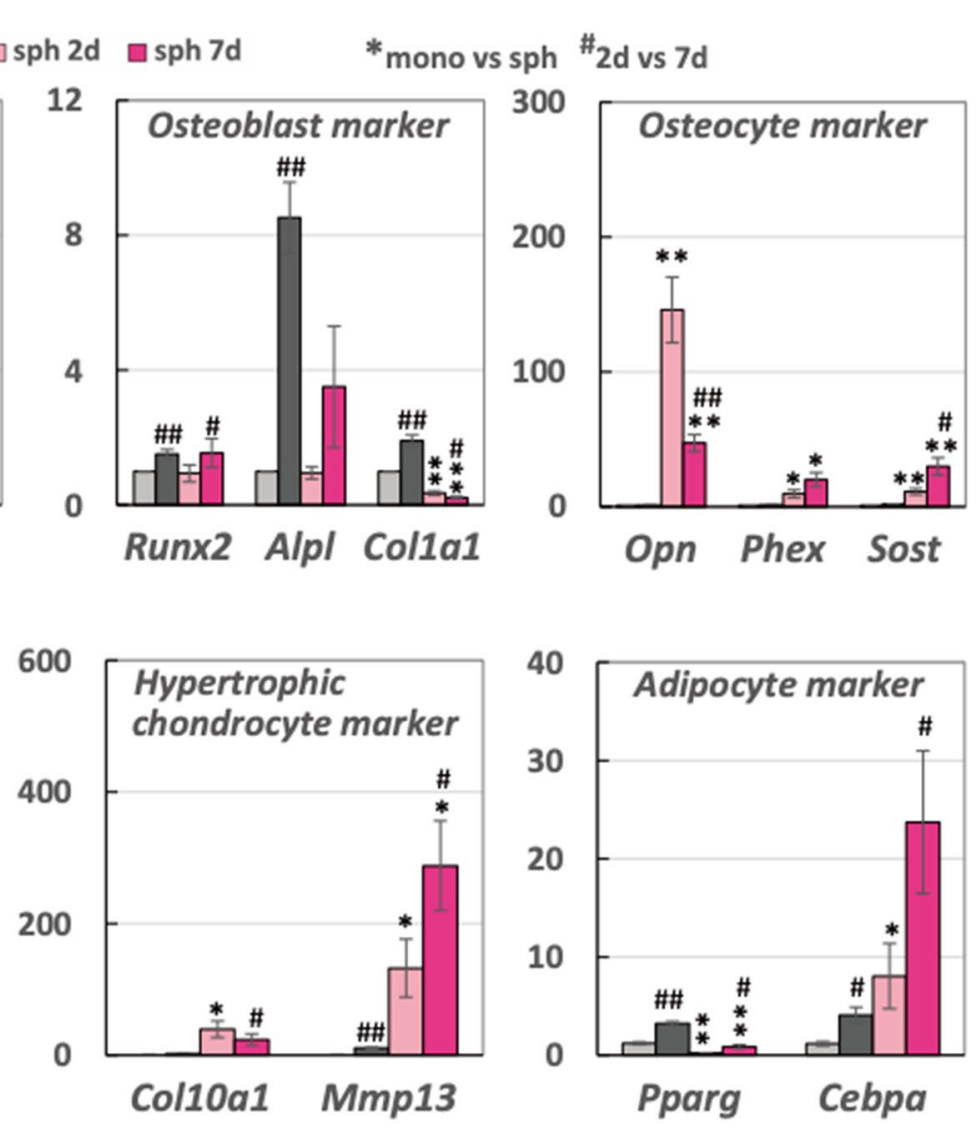

Figure 3. (A) Schematic of the experimental time line for spheroids incubated in the basal medium in the absence of osteogenesis supplements for 7 days. Relative mRNA expressions of (B) stem cell markers (Nanog, Oct4, and Sox2), osteoblast markers (Runx2, Alpl, and Colla1), osteocyte markers (Opn, Phex, and Sost), (C) chondrocyte markers (Sox9 and Acan), hypertrophic chondrocyte marker (Col10a1 and Mmp13), and adipocyte markers (Pparg and Cebpa) were measured by real-time PCR. All the mRNA expressions were normalized to Gapdh expressions while the results were expressed as relative amounts against the expression of monolayer sample $(n=10)$. The bars represent the mean \pm standard error. $P$-value was calculated from Student's $t$-test (mono vs sph: ${ }^{*} p<0.05,{ }^{* *} p<0.005 ; 2$-day vs 7 -day: ${ }^{\#} p<0.05,{ }^{\# \#} p<0.005$ ).

cell markers were significantly up-regulated in both 2-day and 7-day spheroid compared to 2-day monolayer, corresponding to the previous experiments. Particularly, the stem cell markers in the spheroid were further up-regulated after 7-day incubation. Regarding the osteoblast markers, there were significant up-regulations in the monolayer model, whereas those markers in the spheroid exerted a different trend. Especially, the Col1a1 mRNA expressions were significantly down-regulated at both 2-day and 7-day. While there were no significant changes in the osteocyte gene expression in the monolayer model, all the osteocyte markers in the spheroid model were up-regulated at 2-day and 7-day. Particularly, mRNA expression of early osteocyte marker, Opn, in 2-day spheroid was higher than that in 7-day spheroid, whereas the mRNA expressions of matured osteocyte markers involving Phex and Sost in 7-day spheroid was greater than that in 2-day spheroid.

Since the differentiation of hMSCs was not chemically induced into a certain cell lineage in this experiment, we also examined the gene expression change in chondrocyte, hypertrophic chondrocyte, and adipocyte markers in Fig. 3C. Regarding chondrocyte markers, both Sox9 and Acan mRNA expressions were up-regulated in the monolayer condition after 7-day incubation. In the spheroid, however, Acan mRNA expression was greatly suppressed at both 2-day and 7-day incubation although Sox 9 mRNA expression was slightly increased. On the other hand, hypertrophic chondrocyte markers, such as Col10a1 and Mmp13, were greatly up-regulated in the form of spheroid compared to the monolayer. For adipose markers, the adipogenesis master gene, Pparg, was 

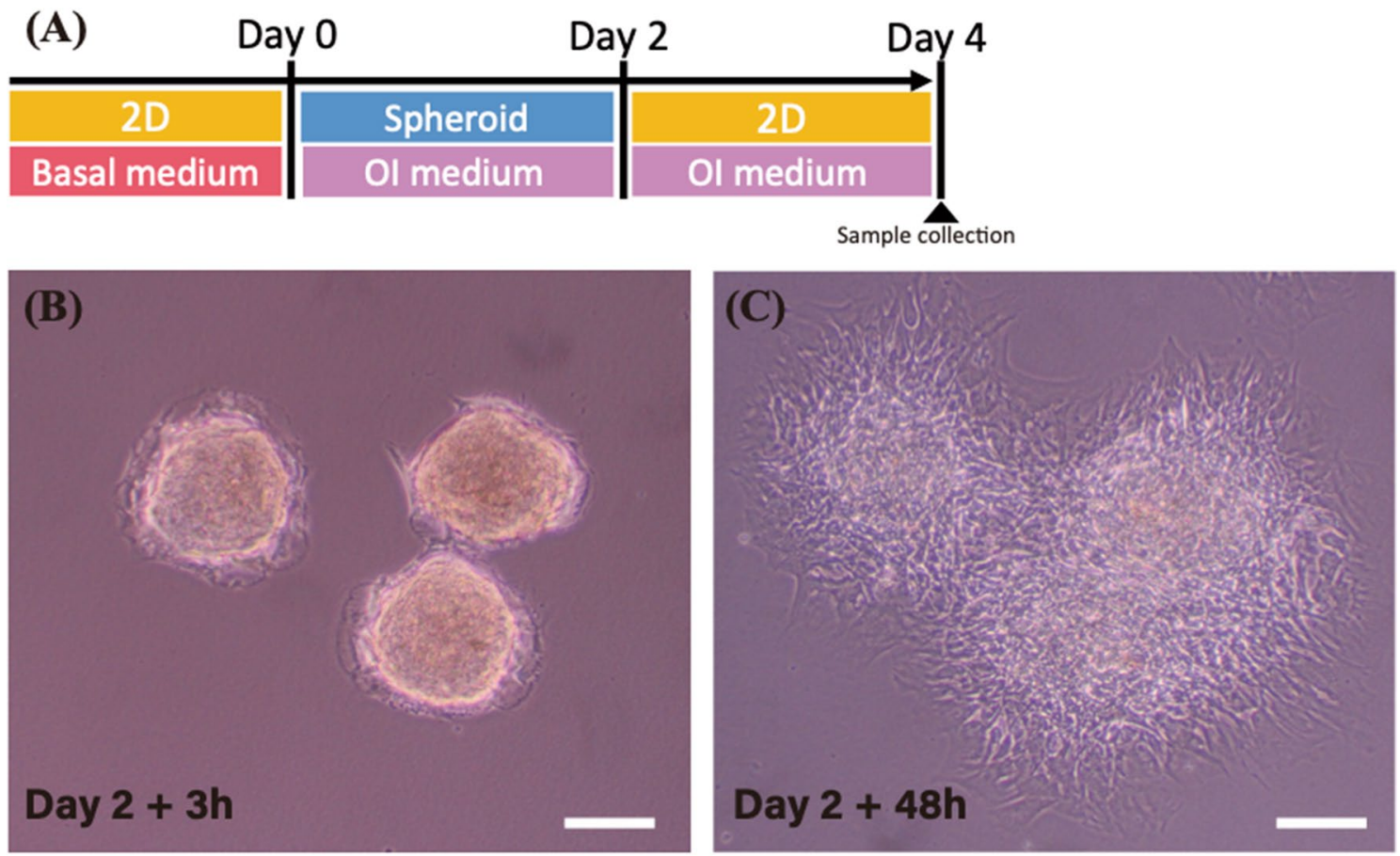

(D)
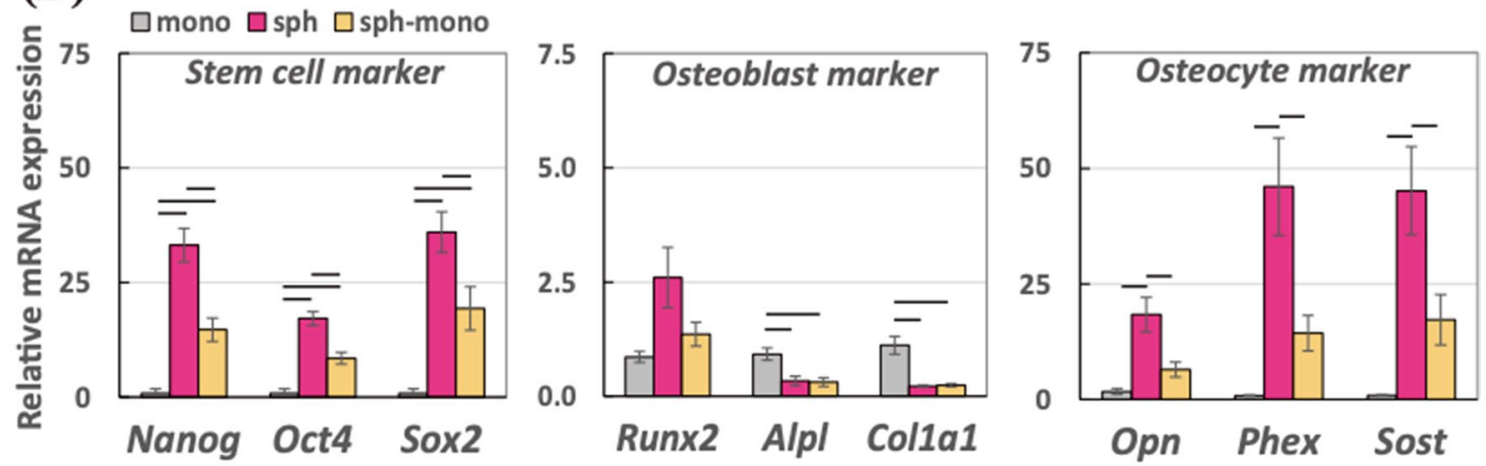

Figure 4. (A) Schematic of the experimental time line to prepare for cells dissociated from the spheroids (sphmono) in the osteogenesis induction medium. Morphology of the spheroids transferred to the normal culture dish after (B) $3 \mathrm{~h}$ and (C) $48 \mathrm{~h}$. The scale bar represents $200 \mu \mathrm{m}$. (D) Relative mRNA expressions of stem cell markers (Nanog, Oct4, and Sox2), osteoblast markers (Runx2, Alpl, and Col1a1), and osteocyte markers (Opn, $P h e x$, and Sost) in monolayer and spheroid incubated for 4 days, and sph-mono were measured by real-time PCR. All the mRNA expressions were normalized to Gapdh expressions while the results were expressed as relative amounts against the expression of monolayer sample $(n=6)$. The graphs represent the mean \pm standard error. Bar indicates the significance between groups, which was calculated from ANOVA with Tukey's HSD posthoc test $(\alpha=0.05)$.

greatly down-regulated in the spheroid although mRNA expression of the metabolic action of insulin marker, Cebpa, was up-regulated in the spheroid. On the other hand, both Pparg and Cebpa mRNA expressions were increased in the monolayer condition after 7-day incubation.

Cell condensed condition in the 3D spheroid structure sustained the osteocyte differentiation. To elucidate the effect of cell condensation achieved in the 3D spheroid structure on the osteocyte differentiation, we introduced another model which the 3D spheroids in the ultra-low attachment dish at 2-day incubation were transferred to a normal culture dish as described in Fig. 4A. Immediate after the spheroid was subcultured on the normal culture dish, the spheroids were initiated to attach onto the dish in Fig. 4B, and then the cells dissociated from the spheroid spread over the dish. After the extra 2-day incubation on the normal culture dish, the spheroid at 2-day lost its 3D structure and became like 2D model in Fig. 4C, termed sph-mono model. We then examined their gene expression levels in the sph-mono model compared to 4-day monolayer and spheroid using real-time PCR in Fig. 4D. Similar to 2-day cultivation results in the previous experiment, 4-day spheroid had higher gene expression levels in the stem cell and osteocyte markers than 4-day monolayer, whereas the mRNA expressions of osteoblast markers such as Alpl and Colla1 in the spheroid were suppressed compared to the monolayer. On the other hand, those up-regulated markers for stem cell and osteocytes were 
down-regulated in the sph-mono model, which indicates that the up-regulations of stem cell and osteocyte markers were depending on the cell condensed condition acquired from the 3D spheroid structure. Regarding osteoblast markers, there was no significant change in the sph-mono model compared to the spheroid.

Cytochalasin D facilitated the osteocyte differentiation in the spheroid by actin depolymerization. To reveal mechanisms for the osteocyte differentiation evoked in the 3D spheroid structure, we utilized an actin polymerization inhibitor, cytochalasin D. During fabrication of spheroid, we added $1 \mu \mathrm{M}$ of cytochalasin D as described in Fig. 5A. Compared to the spheroid with DMSO in Fig. 5B, the size of the spheroid treated with cytochalasin D became bigger as shown in Fig. 5C. In Fig. 5D, after 2-days of incubation, the mean values of the projected area of the spheroid in the presence of DMSO and cytochalasin D became $23,500 \mu \mathrm{m}^{2}$ and 31,300 $\mu \mathrm{m}^{2}$, respectively $(133 \%$ increase; $p<0.005)$. In Fig. 5E, we carried out F-actin/G-actin staining using phalloidin and DNase I to examine the changes in the actin balancing. By the addition of cytochalasin D in the spheroid, the F-actin exhibited among the cells in the spheroid was depolymerized. While both DMSO and Cytochalasin $\mathrm{D}$ exhibited the G-actin expression entirely in the spheroid, the local expression change was not observed. In Fig. 5F, we then conducted real-time PCR to examine the mRNA expression changes in the spheroid by the addition of the inhibitor. Among the stem cell markers, adding cytochalasin D significantly up-regulated Sox 2 mRNA expression; Nanog (1.15-fold change), Oct4 (1.13-fold change), and Sox2 (1.68-fold change; $p<0.05)$. Moreover, osteoblast markers were modulated by cytochalasin D; Runx2 (1.40-fold change; $p<0.005)$, Alpl (2.95-fold change; $p<0.05)$, and Colla1 (0.82-fold change; $p<0.05)$. Particularly, addition of cytochalasin D up-regulated the osteocyte mRNA expressions; Opn (3.80-fold change; $p<0.05)$, Phex (1.15-fold change), Sost (1.24-fold change; $p<0.05)$. Hence, actin depolymerization by cytochalasin $\mathrm{D}$ in the spheroid up-regulated the osteocyte gene expression levels in the spheroid.

\section{Discussion}

Our group has studied the osteocyte differentiation acquired from the $3 \mathrm{D}$ culture system. In the previous studies, we showed that the osteocyte differentiation of pre-osteoblast cells was facilitated in the 3D scaffold-free culture system $^{14-16}$. Although we revealed that cell condensed condition is significant for the pre-osteoblast cells to undergo the osteocyte differentiation, it is still unclear whether or not the osteocyte differentiation can be further evoked from the mesenchymal stem cells to recapitulate the in vivo bone development process. In this study, we assumed that the 3D spheroid culture reconstructed by hMSCs enables to recapitulate the mesenchymal condensation process during the ossification. By using the hMSCs, we fabricated self-organized spheroids and evaluated their osteocyte-likeness in vitro.

Under the OI medium, the hMSC spheroids were chemically induced to differentiate into the osteogenic lineage. As a result of real-time PCR and SOST immunostaining in Figs. 1 and 2, the cells in the spheroid successfully rendered osteocyte-likeness within 2 days while the osteocyte-likeness was further prolonged up to 7 days. Furthermore, the osteoblast markers were greatly suppressed in the spheroid compared to the monolayer condition. The results obtained with the hMSCs in the present study corresponded to the previous studies using the murine pre-osteoblast cells, MC3T3-E $1^{15}$. Moreover, the spheroid also exerted the up-regulation in stem cell markers. The increase in stem cell markers in the spheroid culture was also reported from other studies using stem cells ${ }^{17-19}$. While the osteoblast differentiation in the monolayer condition actively proceeded, the stemness was relatively maintained in the spheroid culture, so that the stem cell gene expressions were relatively up-regulated in the spheroid culture.

We then conducted the experiment with the basal medium for 2 and 7 days in the absence of OI supplements in order to investigate the independent structural effect in the $3 \mathrm{D}$ spheroid culture against the $2 \mathrm{D}$ monolayer culture. Interestingly, the differentiation induction of hMSCs had distinguished trends depending on the cultivation condition. In the monolayer condition, the hMSCs were likely to undertake the osteoblast, chondrocyte, and adipocyte differentiation randomly because of the absence of specific differentiation induction mediums. The result suggested that the monolayer condition seems to be a suitable condition to conduct the osteoblast, chondrocyte, and adipocyte differentiation studies as conducted in other studies for several decades ${ }^{20-22}$. Since no certain chemical supplements were utilized, the hMSCs were thought to differentiate into those cells randomly. While the differentiation processes into osteoblast, chondrocyte, and adipocyte were undertaken on the monolayer, the spheroid condition rendered relatively greater gene expressions in osteocyte and hypertrophic chondrocyte markers as well as stem cell markers. During the endochondral ossification process, it is known that the bone is replaced from the cartilage via hypertrophic chondrocytes ${ }^{23}$. The transient up-regulation of osteocyte and hypertrophic chondrocyte markers in the spheroid model might mimic the certain event of ossification process which is initiated from the mesenchymal condensation in vivo during the bone development process. Regarding the stem cell markers, the increase in stem cell gene expressions was observed, corresponding to the previous experiment using the OI medium in Fig. 1, which indicates that the spheroid culture for the hMSC relatively maintained its stemness compared to the monolayer condition, whereas the cells in the monolayer model are likely to differentiate and commit to some lineages even without certain chemical supplements.

To investigate the cell condensed effect achieved by the 3D spheroid, we transferred the spheroid to the normal culture dish, causing that the $3 \mathrm{D}$ spheroid spread over and became like monolayer condition, termed sph-mono model (the cells dissociated from the 3D spheroid). In terms of gene expression changes in the sph-mono model compared to 4-day spheroid and monolayer, the up-regulated osteocyte mRNA expressions, as well as stemness marker in the spheroids, were diminished after the spheroid lost the condensed condition. The results suggested that the cell condensed condition is essential for the hMSCs to differentiate into osteocytes and further to retain their osteocyte-likeness. In other words, the monolayer condition is not a suitable cell cultural environment for osteocyte-related studies. This is why only a limited number of in vitro osteocytic models were available since 

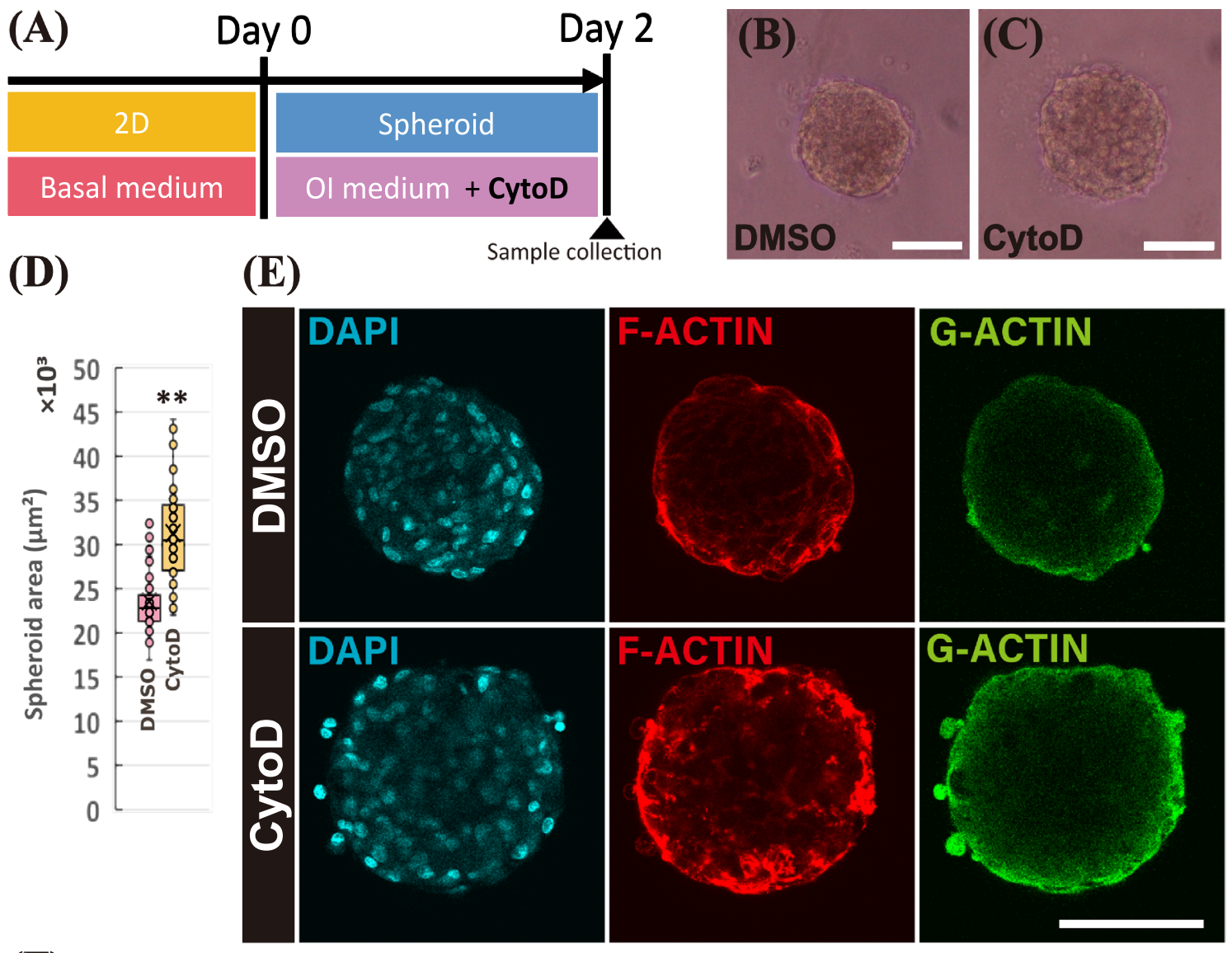

(F) sph+DMSO $\square$ sph+CytoD
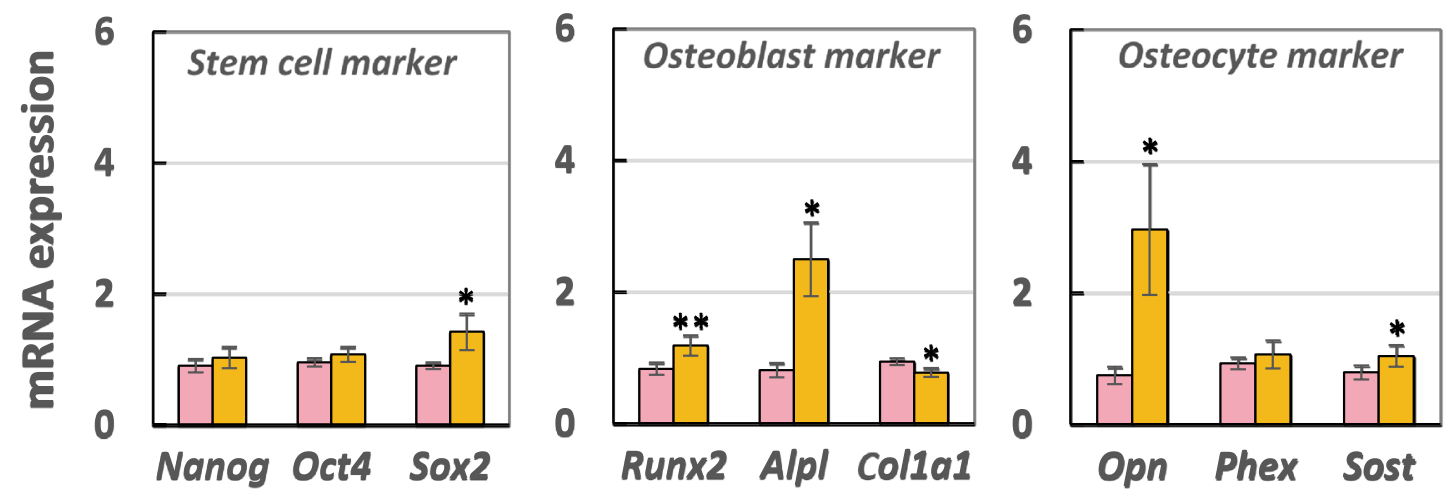

Figure 5. (A) Schematic of the experimental time line to fabricate the spheroid treated with $1 \mu \mathrm{M}$ of cytochalasin D (CytoD). Morphology of the spheroids treated with (B) DMSO and (C) CytoD. The scale bar represents $100 \mu \mathrm{m}$. (D) Mean values of projected area of the spheroids treated with DMSO and CytoD incubated for 2 days. (E) Staining images of spheroid after 2-day incubation; cell nuclei (DAPI in cyan), fibrous actin (F-actin in red), and globular actin (G-actin in green). The scale bar represents $100 \mu \mathrm{m}$. (F) Relative mRNA expressions of stem cell markers (Nanog, Oct4, and Sox2), osteoblast markers (Runx2, Alpl, and Col1a1), and osteocyte markers (Opn, Phex, and Sost) in the spheroid treated with DMSO and CytoD were measured by realtime PCR. All the mRNA expressions were normalized to Gapdh expressions while the results were expressed as relative amounts against the expression of spheroid samples treated with DMSO $(n=9)$. The bars represent the mean \pm standard error. $P$-value was calculated from Student's $t$-test; ${ }^{\star} p<0.05,{ }^{* *} p<0.005$.

many studies attempted to undertake osteocyte differentiation on the monolayer condition. Hence, we hereby highlighted the significance of cell condensation conditions for osteocytogenesis.

In order to reveal the mechanism in the facilitated osteocyte differentiation in the spheroid structure, the involvement of actin balancing was investigated. In this study, cytochalasin D was utilized to inhibit the actin polymerization in the spheroid. As a result, cytochalasin D successfully depolymerized F-actin in the spheroid, 


\section{Intramembranous ossification}
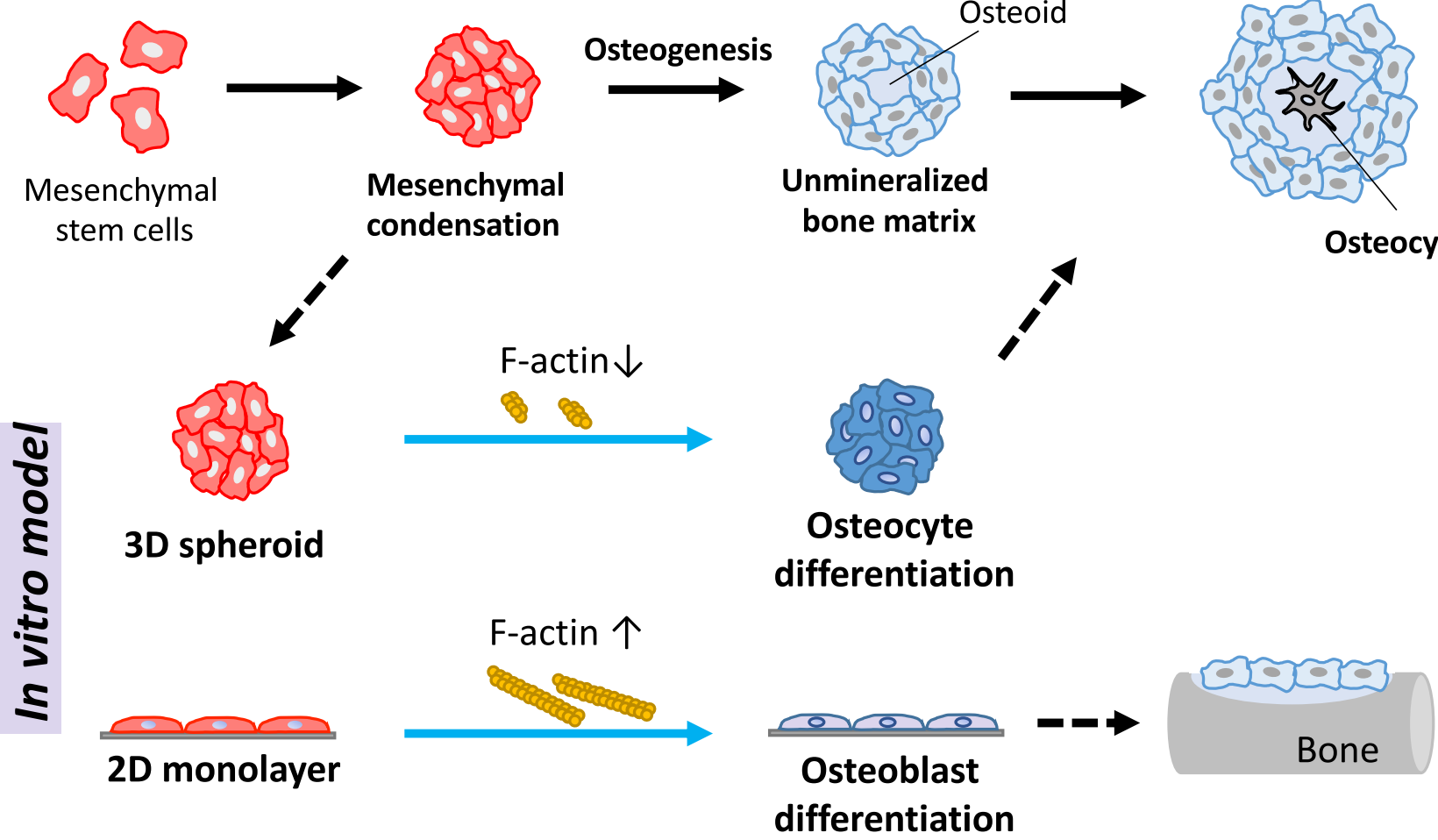

Figure 6. Schematic diagram to highlight the significance of actin depolymerization in the 3D spheroid culture to determine the cell differentiation fate into osteocytes.

resulting in an increase in the size of the spheroid. The prevention of F-actin polymerization by cytochalasin $\mathrm{D}$ eventually promoted osteocyte mRNA expressions in the spheroid. With regard to osteoblast differentiation on the conventional 2D monolayer condition, the actin depolymerization is involved in the osteoblast differentiation of human bone marrow-derived $\mathrm{MSCs}^{24}$. On the other hand, our results first highlighted the involvement of actin depolymerization for the osteocyte differentiation of hMSCs. As illustrated in Fig. 6, this study suggested that the osteocyte differentiation can be achieved by weakened F-actin formation in the spheroid compared to the monolayer, whereas the generation of the tight F-actin on the monolayer model evoked the osteoblast differentiation of hMSCs as well as bone mineralization as shown in other studies ${ }^{24-26}$. It implies the involvement of actin balancing to determine the differentiation fate of MSCs into osteocyte or osteoblast. For the possible mechanism under the change in the actin balancing acquired in the spheroid culture, mitochondrial mobility ${ }^{27}$ or cofilin phosphorylation ${ }^{24,28}$ might alter the actin dynamics, which consequently induced the osteocyte differentiation. Further study will be also needed to examine if the actin dynamics affect other types of cellular differentiation as the actin polymerization is known to involve in the differentiation capability of osteoclast ${ }^{29}$ or megakaryopoiesis and prophatelet formation ${ }^{30}$. Regarding the stemness markers in the spheroid, the actin depolymerization in the stem cells is known to promote their pluripotent states ${ }^{31}$, which corresponds to our results. Based on this inhibitor experiment using the spheroid model, we first showed that the actin dynamics is involved in the osteocyte differentiation induction acquired by the spheroid culture.

As many studies reported, the osteoblasts undergo the bone mineralization process where they are twodimensionally arranged on the bone matrix, which is reconstructed in the conventional 2D monolayer condition for several weeks of cultivation time $\mathrm{e}^{932-34}$. During the in vitro bone formation studies using the 2D model, the osteoblast precursor cells in the confluent monolayer condition eventually forms the 3D dome shape of bone nodules after a long experiment over several weeks ${ }^{11}$. Then, osteocyte-like cells were found in the mineralized nodule ${ }^{13}$. Throughout our present study using the $3 \mathrm{D}$ spheroid culture model, we suggested a new insight that the osteocyte differentiation can be triggered by the 3D cell condensed condition of osteoblast precursor cells within a short period of time at a certain remodeling site. It might indicate that a group of cells in the bone nodule are subjected to the cell condensed condition so that the osteocyte differentiation is acquired after the osteoblast precursor cells are confronted with the cell condensed condition. This explanation might also imply that the osteoblasts in the bone become osteocytes when they encountered the condensed condition at the remodeling site in vivo. For the cells in the condensed condition, they became three-dimensionally surrounded by neighboring cells and their extracellular matrices, so that the formation of actin filaments is thought to be greatly suppressed ${ }^{31}$. We suggest that the osteocytes are not differentiated from the mature osteoblasts, but directly differentiated from the osteoblast precursor cells in the cell condensed condition, as we reported in the previous studies using a 3D 


\begin{tabular}{|l|l|l|l|}
\hline Gene & Forward primer & Reverse primer & Amplicon size (bp) \\
\hline Gapdh & GCTCTCTGCTCCTCCTGTTC & GTTGACTCCGACCTTCACCT & 103 \\
\hline Nanog & GTCCCGGTCAAGAAACAGAA & GCTGAGGTTCAGGATGTTGG & 135 \\
\hline Oct 4 & CCCGAAAGAGAAAGCGAAC & CACTCGGACCACATCCTTCT & 143 \\
\hline Sox 2 & GCCGAGTGGAAACTTTTGTC & GGGCAGCGTGTACTTATCCTT & 192 \\
\hline Run $x 2$ & CCGTCCATCCACTCTACCAC & CCAGAGGCAGAAGTCAGAGG & 120 \\
\hline Alpl & GGAGATGGGATGGGTGTCT & CCACGAAGGGGAACTTGTC & 112 \\
\hline Colla 1 & GACGAAGACATCCCACCAAT & CATCGCACAACACCTTGC & 121 \\
\hline Opn & GCCGAGGTGATAGTGTGGTT & GCTTTCCATGTGTGAGGTGAT & 113 \\
\hline Phex & GACTTTGCTGAGGGAGAGCA & CGCCACCCTTTCTCAAATAA & 132 \\
\hline Sost & CAGCCTTCCGTGTAGTGGAG & CGGTTCATGGTCTTGTTGTTC & 127 \\
\hline Sox 9 & TGGAGACTTCTGAACGAGAGC & CGTTCTTCACCGACTTCCTC & 127 \\
\hline Acan & ACTCGCTGACCTTTGAGGAG & GGCTCACAATGGGGTATCTG & 156 \\
\hline Col10a1 & CTTCTGCACTGCTCATCTGG & GTGGGCATTTGGTATCGTTC & 179 \\
\hline Mmp13 & GCTGCCTTCCTCTTCTTGAG & CTCAGTCATGGAGCTTGCTG & 180 \\
\hline Pparg & GAAGGATGCAAGGGTTTCTTC & CCAACAGCTTCTCCTTCTCG & 199 \\
\hline Cespa & GGGACCGGAGTTATGACAAG & GGCAAGTATCCGAGCAAAAC & 174 \\
\hline
\end{tabular}

Table 1. Primer list.

culture model reconstructed by osteoblast precursor cells ${ }^{14-16}$. Consequently, the cell condensed condition plays a significant role to determine the cellular differentiation fate of MSCs into the osteocytes.

In this study, the 3D spheroid reconstructed by MSCs under the OI medium exerted the osteocyte-likeness within 2 days. Notably, we first suggested the alteration of the actin balancing induced by cell condensed condition in the spheroid culture to determine the MSC differentiation fate to osteocytes. Moreover, we elucdiated the structural effect on MSC differentiation, which provides a suitable strategy for the cultural model depending on certain cell lineage studies. Hence, the present 3D spheroid culture model has the potential to represent the osteocyte and further to recapitulate the in vitro ossification process, becoming a new in vitro model as "bone organoid".

\section{Methods}

Cell culture. Human bone marrow-derived MSCs (passage 2) were acquired from PromoCell (Germany) and RIKEN BRC (Japan). In this study, we utilized several lines obtained from 5 different donors (PromoCell: C-12974; RIKEN RBC: MSC-R37, MSC-R43, MSC-R44, and MSC-R50.). The cells were maintained in the basal medium which is low glucose-Dulbecco's Modified Eagle Medium (Gibco, USA) supplemented with 10\% fetal bovine serum (Gibco, USA) and $1 \%$ antibiotic-antimycotic (Gibco, USA) solution in a humidified incubator at $37^{\circ} \mathrm{C}$ under $5 \% \mathrm{CO}_{2}$ condition. We carried out the cell passaging every $2-3$ days when the cell confluency became $80-90 \%$. For experiments, the cells from passage 4 up to 12 were used. To prepare an osteogenic induction (OI) medium, we utilized high glucose-Dulbecco's Modified Eagle Medium (Gibco, USA) containing $50 \mu \mathrm{M}$ ascorbic acid (Wako, Japan), $10 \mathrm{mM} \beta$-glycerophosphate (Sigma, USA), and $100 \mathrm{nM}$ dexamethasone (Nacalai Tesque, Japan). To prepare for 2D monolayer samples, 200,000 cells were subcultured on a $35 \mathrm{~mm}$ diameter culture dish to become confluent after 2-day incubation. This study was approved by the Ethics Committee of Institute for Frontier Life and Medical Sciences, Kyoto University (Clinical Protocol No. 89).

Fabrication of spheroids. Based on the previous study ${ }^{15}$, we fabricated self-organized spheroids using hMSCs on the Nunclon Sphera 96-well round-bottom plate (ThermoFisher, USA). 2500 cells were subcultured on the round-bottom plate to fabricate the spheroids and incubated for 2, 4 or 7 days in the presence or absence of the OI medium depending on the aims of each experiments.

Real time-PCR. To measure mRNA expressions in the spheroid and monolayer reconstructed by hMSCs, we conducted real-time PCR. After the samples were rinsed with PBS once, they are immediately lysed with Isogen II (Nippon Gene, Japan). According to the manufacturer's protocol, RNA extraction and cDNA synthesis were carried out using PureLink RNA Mini kit (Invitrogen, USA) and Transcriptor Universal cDNA Master (Roche, Switzerland), respectively. For the real-time PCR, we used PowerUp SYBR Green Master Mix (ThermoFisher, USA) while all the PCR primers were designed as shown in Table 1. Nanog homeobox (Nanog), POU class 5 homeobox (Oct4), and SRY-box transcription factor 2 (Sox2) were used as undifferentiated stem cell markers. For osteoblast markers, we examined mRNA expressions of runt-related transcription factor 2 (Runx2), alkaline phosphatase ( $\mathrm{Alpl}$ ), and type I collagen, alpha 1 chain (Col1a1). To distinguish osteocytes, we utilized osteopontin $(O p n)$, phosphate regulating endopeptidase homolog X-linked (Phex), and sclerostin (Sost). While SRY-box transcription factor 9 (Sox9) and aggrecan (Acan) were used as chondrocyte markers, type X collagen, alpha 1 chain (Col10al) and matrix metallopeptidase 13 ( Mmp13) were examined as hypertrophic chondrocyte markers. For adipocyte markers, we utilized peroxisome proliferator activated receptor gamma 
(Pparg) and CCAAT enhancer binding protein alpha (Cebpa). All the mRNA expressions were normalized to a reference gene, glyceraldehyde-phosphate dehydrogenase (Gapdh) and measured by using the $2^{-\Delta \Delta C T}$ method.

Staining. The spheroids were collected and fixed in $4 \%$ paraformaldehyde (PFA). After fixation, the samples were permeabilized with $0.1 \%$ triton X-100 in PBS for 30 min and washed with PBS 3 times. For immunostaining, non-specific binding during staining was blocked with PBS containing $4 \%$ bovine serum albumin at room temperature for $1 \mathrm{~h}$. Then, we added the anti-SOST antibody (Sigma, USA) and incubated at room temperature for $1 \mathrm{~h}$. After washing with PBS 3 times, the samples were treated with the Alexa Flour 488 secondary antibody (Invitrogen, USA), Alexa Fluor 546 Phalloidin (Invitrogen, USA), and DAPI (Sigma, USA) at room temperature for $1 \mathrm{~h}$. After washing with PBS 3 times, the samples were observed by the FLUOVIEW FV3000 (Olympus, Japan). For G-actin (globular) and F-actin (fibrous) staining, the samples were treated with the Deoxyribonuclease I (DNase I), Alexa Fluor 488 conjugate (Invitrogen, USA) and Alexa Fluor 546 Phalloidin (Invitrogen, USA), respectively, and counterstained with DAPI (Sigma, USA) at room temperature for $15 \mathrm{~min}$. After washing with PBS 3 times, the stained samples were mounted on the glass slides for visualization.

Statistical analysis. The statistical significance was assessed by Student's t-test, F-test, or ANOVA followed by Tukey's honestly significant difference (HSD) post-hoc test (with $\alpha=0.05$ ). P-values less than 0.05 were considered to be significant. The statistical analysis was implemented in Excel and Python.

Received: 6 April 2021; Accepted: 14 June 2021

Published online: 24 June 2021

\section{References}

1. Adachi, T. et al. Osteocyte calcium signaling response to bone matrix deformation. J. Biomech. 42, 2507-2512 (2009).

2. Robling, A. G. \& Bonewald, L. F. The osteocyte: New insights. Annu. Rev. Physiol. 82, 485-506 (2020).

3. Caplan, A. I. Mesenchymal stem cells. J. Orthop. Res. 9, 641-650 (1991).

4. Pittenger, M. F. et al. Multilineage potential of adult human mesenchymal stem cells. Science 284, 143-147 (1999).

5. Pittenger, M. F. et al. Mesenchymal stem cell perspective: Cell biology to clinical progress. NPJ Regenerat. Med. 4, 22 (2019).

6. Parfitt, A. M. The bone remodeling compartment: A circulatory function for bone lining cells. J. Bone Miner. Res. 16, 1583-1585 (2001).

7. Rutkovskiy, A., Stensløkken, K.-O. \& Vaage, I. J. Osteoblast differentiation at a glance. Med. Sci. Monit. Basic Res. 22, 95-106 (2016).

8. Manolagas, S. C. Birth and death of bone cells: Basic regulatory mechanisms and implications for the pathogenesis and treatment of osteoporosis. Endocr. Rev. 21, 115-137 (2000).

9. Coelho, M. J. \& Fernandes, M. H. Human bone cell cultures in biocompatibility testing. Part II: Effect of ascorbic acid, $\beta$-glycerophosphate and dexamethasone on osteoblastic differentiation. Biomaterials 21, 1095-1102 (2000).

10. Buttery, L. D. K. et al. Differentiation of osteoblasts and in Vitro bone formation from murine embryonic stem cells. Tissue Eng. 7, 89-99 (2001).

11. Bhargava, U., Bar-Lev, M., Bellows, C. G. \& Aubin, J. E. Ultrastructural analysis of bone nodules formed in vitro by isolated fetal rat calvaria cells. Bone $9,155-163$ (1988).

12. Mechiche Alami, S., Gangloff, S. C., Laurent-Maquin, D., Wang, Y. \& Kerdjoudj, H. Concise review. In vitro formation of bone: Like nodules sheds light on the application of stem cells for bone regeneration. Stem Cells Transl. Med. 5, 1587-1593 (2016).

13. Kawai, S. et al. In vitro bone-like nodules generated from patient-derived iPSCs recapitulate pathological bone phenotypes. Nat. Biomed. Eng. 3, 558-570 (2019).

14. Kim, J. \& Adachi, T. Cell condensation triggers the differentiation of osteoblast precursor cells to osteocyte-like cells. Front. Bioeng. Biotechnol. 7, 288 (2019).

15. Kim, J., Kigami, H. \& Adachi, T. Characterization of self-organized osteocytic spheroids using mouse osteoblast-like cells. J. Biomech. Sci. Eng. 15, 1-8 (2020).

16. Kim, J. \& Adachi, T. Modulation of Sost gene expression under hypoxia in 3D scaffold-free osteocytic tissue. Tissue Eng. A. https:// doi.org/10.1089/ten.tea.2020.0228 (2020).

17. Cheng, N. C., Wang, S. \& Young, T. H. The influence of spheroid formation of human adipose-derived stem cells on chitosan films on stemness and differentiation capabilities. Biomaterials 33, 1748-1758 (2012).

18. Guo, L., Zhou, Y., Wang, S. \& Wu, Y. Epigenetic changes of mesenchymal stem cells in three-dimensional (3D) spheroids. J. Cell Mol. Med. 18, 2009-2019 (2014).

19. Zhou, Y., Chen, H., Li, H. \& Wu, Y. 3D culture increases pluripotent gene expression in mesenchymal stem cells through relaxation of cytoskeleton tension. J. Cell Mol. Med. 21, 1073-1084 (2017).

20. Hanna, H., Mir, L. M. \& Andre, F. M. In vitro osteoblastic differentiation of mesenchymal stem cells generates cell layers with distinct properties. Stem Cell Res. Ther. 9, 1-11 (2018).

21. Shirasawa, S. et al. In vitro chondrogenesis of human synovium-derived mesenchymal stem cells: Optimal condition and comparison with bone marrow-derived cells. J. Cell. Biochem. 97, 84-97 (2006).

22. Janderová, L., McNeil, M., Murrell, A. N., Mynatt, R. L. \& Smith, S. R. Human mesenchymal stem cells as an in vitro model for human adipogenesis. Obes. Res. 11, 65-74 (2003).

23. Aghajanian, P. \& Mohan, S. The art of building bone: Emerging role of chondrocyte-to-osteoblast transdifferentiation in endochondral ossification. Bone Res. 6, 1-10 (2018).

24. Chen, L. et al. Inhibiting actin depolymerization enhances osteoblast differentiation and bone formation in human stromal stem cells. Stem Cell Res. 15, 281-289 (2015).

25. Rodríguez, J. P., González, M., Ríos, S. \& Cambiazo, V. Cytoskeletal organization of human mesenchymal stem cells (MSC) changes during their osteogenic differentiation. J. Cell. Biochem. 93, 721-731 (2004).

26. Sonowal, H., Kumar, A., Bhattacharyya, J., Gogoi, P. K. \& Jaganathan, B. G. Inhibition of actin polymerization decreases osteogeneic differentiation of mesenchymal stem cells through p38 MAPK pathway. J. Biomed. Sci. 20, 1-10 (2013).

27. Zhang, Y. et al. iPSC-MSCs with high intrinsic MIRO1 and Sensitivity to TNF- $\alpha$ yield efficacious mitochondrial transfer to rescue anthracycline-induced cardiomyopathy. Stem Cell Rep. 7, 736-749 (2016).

28. Wioland, H. et al. Actin filament oxidation by MICAL1 suppresses protections from cofilin-induced disassembly. EMBO Rep. 22, 965 (2021)

29. Destaing, O. et al. The tyrosine kinase activity of c-Src regulates actin dynamics and organization of podosomes in osteoclasts. Mol. Biol. Cell 19, 394-404 (2008). 
30. Ye, J. Y. et al. Serotonin enhances megakaryopoiesis and proplatelet formation via p-Erk1/2 and F-actin reorganization. Stem Cells 32, 2973-2986 (2014).

31. Yu, L. et al. Low cell-matrix adhesion reveals two subtypes of human pluripotent stem cells. Stem Cell Rep. 11, 142-156 (2018).

32. Suh, W. K. et al. The immune regulatory protein B7-H3 promotes osteoblast differentiation and bone mineralization. Proc. Natl. Acad. Sci. USA 101, 12969-12973 (2004).

33. Li, X. et al. Dkk2 has a role in terminal osteoblast differentiation and mineralized matrix formation. Nat. Genet. 37, 945-952 (2005).

34. Gordon, J. A. R. et al. Bone sialoprotein expression enhances osteoblast differentiation and matrix mineralization in vitro. Bone 41, 462-473 (2007).

\section{Acknowledgements}

We would like to thank Junko Sunaga for providing technical supports. This work was supported by the Japan Society for the Promotion of Science (JSPS) KAKENHI (20H00659, 20K20181, and 19K23604), Japan Agency for Medical Research and Development (AMED-CREST) (JP20gm0810003 and JP19bm0804006), and by Nakatani Foundation for Advancement of Measuring Technologies in Biomedical Engineering (Encouragement of Research).

\section{Author contributions}

J.K. and T.A. conceived and supervised the study; J.K. designed and performed the experiments; J.K. analyzed the data; J.K. wrote the manuscript; T.A. made the manuscript revision.

\section{Competing interests}

The authors declare no competing interests.

\section{Additional information}

Correspondence and requests for materials should be addressed to J.K.

Reprints and permissions information is available at www.nature.com/reprints.

Publisher's note Springer Nature remains neutral with regard to jurisdictional claims in published maps and institutional affiliations.

(c) Open Access This article is licensed under a Creative Commons Attribution 4.0 International License, which permits use, sharing, adaptation, distribution and reproduction in any medium or format, as long as you give appropriate credit to the original author(s) and the source, provide a link to the Creative Commons licence, and indicate if changes were made. The images or other third party material in this article are included in the article's Creative Commons licence, unless indicated otherwise in a credit line to the material. If material is not included in the article's Creative Commons licence and your intended use is not permitted by statutory regulation or exceeds the permitted use, you will need to obtain permission directly from the copyright holder. To view a copy of this licence, visit http://creativecommons.org/licenses/by/4.0/.

(C) The Author(s) 2021 